Sarah Sturrock, Emma Williams and Anne Greenough*

\title{
Antenatal and perinatal outcomes of refugees in high income countries
}

https://doi.org/10.1515/jpm-2020-0389

Received August 14, 2020; accepted August 17, 2020; published online September 1, 2020

\section{Abstract}

Objectives: The World Health Organisation (WHO) has highlighted a marked trend for worse pregnancy-related indicators in migrants, such as maternal and neonatal morbidity and mortality, poor mental health and suboptimal care. The aim of this study was to determine whether such adverse outcomes occurred in refugees who moved to high income countries by comparing their antenatal and perinatal outcomes to those of nonimmigrant women.

Methods: A literature search was undertaken. Embase and Medline databases were searched using Ovid. Search terms included "refugee”, "pregnan*” or "neonat*”, and "outcome".

Results: The search yielded 194 papers, 23 were included in the final analysis. All the papers included were either retrospective cohort or cross-sectional studies. The refugees studied originated from a wide variety of source countries, including Eritrea, Somalia, Afghanistan, Iraq, and Syria. Refugee women were more likely to be socially disadvantaged, but less likely to smoke or take illegal drugs during pregnancy. Refugee women were more likely to have poor, late, or no attendance at antenatal care. Miscarriages and stillbirth were more common amongst refugee women than non-refugees. Perinatal mortality was higher among refugees.

*Corresponding author: Professor Anne Greenough, Women and Children's Health, School of Life Course Sciences, Faculty of Life Sciences and Medicine, King's College London, London, UK; The Asthma UK Centre in Allergic Mechanisms of Asthma, Kings College London, London, UK; Neonatal Intensive Care Centre, King's College Hospital NHS Foundation Trust, 4th Floor Golden Jubilee Wing, Denmark Hill, London, SE5 9RS, UK, Phone: +44 0203299 3037, Fax: +44 0203299 8284, E-mail: anne.greenough@kcl.ac.uk. https://orcid.org/0000-00028672-5349

Sarah Sturrock and Emma Williams, Women and Children's Health, School of Life Course Sciences, Faculty of Life Sciences and Medicine, King's College London, London, UK. https://orcid.org/0000-00016480-468X (E. Williams)
Conclusions: Despite better health care services in high income countries, refugee mothers still had worse outcomes. This may be explained by their late or lack of attendance to antenatal care.

Keywords: antenatal outcomes; miscarriages; perinatal mortality; refugees; still birth.

\section{Introduction}

The current refugee crisis has led to the highest levels of displacement on record according to the United Nations High Commissioner for Refugees (UNHCR); 70.8 million people worldwide have been forcibly displaced, 25.9 million of whom are refugees. Fifty-seven percent originate from three countries: Syria, Afghanistan and South Sudan [1]. Although women represent almost half of refugee populations, they are not given the same opportunities as male refugees. Only $0.4 \%$ of funding to fragile states went to women's groups or women's ministries from 2012 to 2013 [2] and refugee girls represented only $30 \%$ of refugee children enrolled in secondary school [3].

Six to $14 \%$ of women in refugee populations would be expected to be pregnant [3]. The United Nations (UN) has stated that $60 \%$ of preventable maternal deaths take place in humanitarian settings [2], and the World Health Organization (WHO) highlighted a "marked trend for worse pregnancy-related indicators in migrants", such as increased maternal and neonatal morbidity and mortality, poorer mental health, and more frequently suboptimal care [4]. Despite the challenges of studying maternal and neonatal outcomes within refugee camps, available data suggest that outcomes for this population are poor, with a stillbirth rate as high as 9.4 per 1,000 total births in the Zaatari camp in Jordan [5].

Over 3,000,000 refugees have resettled in highincome countries [6], where healthcare provision would be expected to be better than in low income countries. Good quality antenatal and perinatal healthcare in high income countries might mitigate some of the risks associated with being a refugee mother. The aim of this study was to undertake a literature review to determine if, in high income countries, the antenatal, perinatal and neonatal outcomes of refugee women differed from those of non- 
immigrant women. These results would enable better targeting of care if differences were highlighted.

\section{Materials and methods}

Embase and Medline databases were searched using Ovid. Search terms included "refugee", "pregnan"” or "neonat”", and "outcome". Articles were included if they were published in peer-reviewed journals in English, and compared neonatal outcomes of infants born to refugee mothers who had migrated to high-income countries (using the World Bank definition [7]) with neonatal outcomes in the native population, or non-refugee migrants resident in the same country. Articles were excluded if they did not compare refugee outcomes to non-refugee outcomes, if they were not published in English or if they studied refugee women who had migrated to low- or middle-income countries. Bibliographies of relevant articles were searched for additional papers meeting the inclusion and exclusion criteria.

\section{Ethical approval}

Ethical approval was not required for this study as it was a literature review.

\section{Results}

The search yielded 194 papers, 55 of which were identified as duplicates. Titles and abstracts were screened and 91 papers were excluded. Of these, 52 were excluded for studying an inappropriate population, 19 due to the outcome studied, 14 due to publication type and six due to study design. A total of 48 papers were read in full, of which 23 were included in the final analysis. All the papers included were either retrospective cohort or cross-sectional studies.

\section{Demographic factors}

The refugees studied originated from a wide variety of source countries, including Eritrea, Somalia, Afghanistan, Iraq, and Syria [8-12].

In one study, refugee mothers were found to have fewer medical risk factors pre-pregnancy such as hypertension or diabetes on retrospective review of medical records [8]. A Swedish study, however, found self-rated pregnancy health in refugees, asylum seekers and undocumented migrants to be poorer than in native-born women [13]. In another study, refugee women were more likely to be socially disadvantaged [10], but less likely to smoke or take illegal drugs during pregnancy than US-born women [8].

The body mass index (BMI) of presumed refugee women varied according to country of origin and comparator population. Two studies, both of which compared refugee women from Africa to native-born women in the USA and in Israel, found no significant difference in pre-pregnancy BMIs between the groups $[8,14]$. The study in Israel also recorded weight gain during pregnancy, which was lower in the refugee population [14]. Two studies compared refugee women to nonhumanitarian migrants in Australia. One study, which included refugee women from Africa, Asia, and the Middle East found that refugees had a higher pre-pregnancy BMI than non-humanitarian migrants [11]. In the other, which assessed only refugees from Africa they were found to have a lower pre-pregnancy BMI than non-humanitarian migrants from the same continent [15].

Most studies found that refugee mothers were younger than non-refugee mothers [10-12, 14, 16, 17], although one study found that refugees from West Asia were less likely to be under 20 years of age than non-refugees originating from the same region [12], and another reported that refugees had an older mean age than US-born women [18]. The mean age of the US-born women, however, was 25.7 which was similar to the mean age (25.9) of refugees in another study [14] (See Table 1).

\section{Maternal risk factors}

The majority of studies found that refugee women were more likely to be multiparous or of higher parity than native women $[8,10,17,19,20]$ and other migrant women $[11,15]$. One study found that refugee mothers were more likely to be nulliparous than non-refugees [14], but the number of refugees included was small.

Refugee women were more likely to have poor, late, or no attendance at antenatal care $[8,11,12,16,18]$, including no ultrasound screening [13]. One study found that $20 \%$ of refugee women had no antenatal care [17]. Refugee women were also more likely to give birth before arriving at hospital [11].

Refugee women were reported to be less likely to have high-risk pregnancy conditions [21] such as pre-eclampsia $[13,17,19]$, gestational diabetes [11, 12], and antepartum haemorrhage [11]. One study, found no significant difference in rates of gestational diabetes or pregnancy induced hypertension between refugees and non-refugees, but only 13 refugee women in the study had gestational diabetes [16]. One study reported an increased risk of gestational diabetes amongst refugee women, although this was the only study comparing refugee women to Swedish-born women [13]. There was no significant difference in the risk of gestational diabetes when refugee women were compared to 
undocumented migrants and asylum seekers [13]. In Canada, amongst women with gestational diabetes, refugees were more likely to have endocrinology visits only late in pregnancy and were at increased risk of new-onset diabetes after pregnancy compared to native-born women [19].

\section{Gestational age at delivery}

Most studies found that refugee women were less likely to have a preterm delivery than other migrant groups and native-born women in the USA, Canada, Australia or Sweden [8, 11-13, 18-20]. Two studies, however, found refugees were more likely to have a preterm delivery than native-born women in Israel and Greece respectively [14, 17]. A study of expatriated and non-displaced women in Croatia found that in the first 2 years of the study (1990-91), expatriated women were more likely to deliver prematurely, but this difference was no longer significant in the final year (1992) of the three-year study leading up to the Bosnian War [22]. One study compared primary refugees (those who had come directly from their country of origin) and secondary refugees (those who had lived elsewhere before arriving in their host country) to primary and secondary migrants. It demonstrated that the primary refugees had similar rates of very and moderate preterm birth, whereas the secondary refugees had a higher risk of preterm birth than secondary non-refugee migrants [23]. Two studies found no significant difference in gestational age at delivery between refugees and non-refugees $[9,16]$, and two studies found that refugee women were more likely to deliver post-term as well as pre-term [11, 12].

\section{Mode of delivery}

Most studies found that refugee women were more likely to deliver vaginally $[8,9]$ and less likely to have an instrumental [10-12, 15] or operative birth [9]. Refugee women were also less like to have obstructed [9] or induced [8, 10, 11] labour, except in a study comparing Southeast Asian refugees to other Southeast Asian migrants [12]. In one Finnish study comparing Kurdish and Somalian refugee women to Russian migrant women, Somali women were more likely to have delivery-related complications such as obstructed labour, fetal stress, perineal laceration, and postpartum haemorrhage, although the difference was only significant after adjustment for confounding factors [9]. Postnatal complications were found to be higher in asylum-seeking women compared with German residents, except in the 12-20 years old age group [21]. One study found a higher rate of instrumental births in refugee women, although this was in comparison to Swedish-born women as opposed to another migrant group [13]. It was reported in two studies that refugee mothers were more likely to have meconium in their liquor $[8,14]$.

Seven studies reported that refugee women were less likely to undergo a Caesarean section [8, 10-12, 15, 21, 24], except refugee women aged between 41 and 50 years [21]. Five studies reported that presumed refugees (migrants from a humanitarian source country) were more likely to have Caesarean sections than non-refugees $[13,14,17,20$, 25]. However, of these studies, one compared refugee women to economic migrants [25], three to native-born women $[13,14,17]$ and one to non-refugee women including other migrants and native-born women [20]. Of the seven studies reporting that refugee women were less likely to undergo Caesarean delivery, four used comparison groups of other migrants $[11,12,15,24]$. One study found no significant difference in the rate of Caesarean deliveries when comparing Kurdish and Somali women to the general Finnish population [9] and another also found no significant difference with the exception of refugee multiparous women, who were more likely to have Caesarean sections than non-refugee multiparous women [16].

\section{Antenatal outcomes}

Stillbirth appears commoner amongst refugee women than non-refugees [11, 13, 17, 20, 21]. No significant differences were found in the rates of fetal abnormalities in two studies [11, 12], although another study reported lower rates of congenital anomalies than in infants of native-born women [20].

\section{Neonatal and perinatal mortality}

A study in Croatia of expatriated (women from occupied areas of Croatia and Bosnia and Herzegovina) and nondisplaced women (from free areas of Croatia) found that the perinatal mortality was significantly higher amongst expatriated women [22]. Similarly, a study in the Netherlands reported that presumed refugees and asylum seekers had a higher perinatal mortality rate, which was only partly explained by prematurity [26]. A study in Canada also found higher perinatal mortality amongst refugees [20]. Subsequent mortality has been rarely reported, but one study found no significant differences in the neonatal mortality rate when comparing refugee 
populations to other non-refugee immigrants [20] and another no significant difference in neonatal death before discharge when comparing presumed refugees (migrants from humanitarian source countries [HSCs]) to other migrants [11].

\section{Neonatal morbidity}

The evidence regarding fetal growth restriction and low birthweight is conflicting. Refugee women were found to be less likely to have low birthweight or growth restricted infants in three studies $[8,11,12]$, two of which compared women from HSCs (presumed refugees) to non-refugee migrants from the same subcontinent [11, 12]. Two studies, however, reported no significant difference in the rates of low birthweight $[14,16]$, although both of these studies included native-born women in their comparison group. Three other studies reported higher rates of low birthweight, fetal growth restriction and small for gestational age infants amongst refugee/asylum seeking populations $[13,17,22]$, although two of these studies used an exclusively native-born comparison group.

There have been few studies assessing the requirement for neonatal intensive care unit (NICU) admission and the evidence is conflicting. One study found no significant difference in the rate of admission to the NICU between infants of refugee and non-refugee migrant women [24]. Two studies reported that babies from HSCs were less likely to require NICU admission than non-refugee migrant women from the same subcontinent [11, 12], but two other studies reported that babies born to refugees were more likely to require NICU admission, one used native-born women as the comparator group [14] the other a nonrefugee comparator group including other migrants and native-born women [20].

One study used a composite measure of adverse outcome, including stillbirth, preterm birth, growth restriction, low birthweight, NICU admission, and congenital abnormality and found no significant association between refugee status and adverse outcome [27]. Other studies, however, found that infants born to refugees were more likely to have respiratory conditions, congenital anomalies, hospital readmission due to inadequate weight gain [28] or require ventilatory support, intravenous fluids, or blood transfusions [29]. Amongst babies born to women with gestational diabetes, those born to refugees were less likely to have respiratory distress or jaundice [19] and be macrosomic than those born to non-refugees [19]. One study found no significant difference in Apgar scores at 5 min [11] but three studies found that infants of refugees and asylum seekers were more likely to have low Apgar scores [13, 14, 17]. Furthermore, refugee infants have been reported to have higher rates of hypoxic ischaemic encephalopathy than other immigrants [29]. Infants born to refugees in the USA were almost nine times more likely to have congenital toxoplasmosis even after adjustment for the mother's educational level, gravidity, and country of birth than other migrants or native born women [30].

\section{Discussion}

This review has highlighted that that refugee women were more likely to have poor, late or no antenatal care $[8,11-13$, 16-18]. Furthermore, despite the lack of medical risk factors, many studies found that fetuses born to refugee women were more likely to be stillborn $[11,1,17,20,21]$, and some studies reporting their infants were more likely to have low Apgar scores $[13,14,17]$ and had higher morbidity [27-30]. This was despite studies reporting that refugee women started pregnancy as lower-risk patients (being younger and with fewer comorbidities) [8, 10-12, 14-17]. In addition, they had medically lower-risk pregnancies with fewer pregnancy-related conditions [11-13, 17, 19, 21] and were less likely to have preterm $[8,11-14,18-20]$ or assisted and operative deliveries [8-12, 15, 21, 24].

Previous studies comparing all immigrant women (including economic migrants) to native women in highincome countries found that rates of pregnancy-related maternal conditions, prematurity and neonatal morbidity were all lower in migrants [31,32]. This contrasts to our findings that refugee women and their infants had poorer outcomes with regards to stillbirth, low Apgar scores, and neonatal morbidity. This may indicate that there are risks experienced by refugee women that are not faced by economic migrants to high-income countries. A systematic review found that outcomes were worse for neonates born to immigrant mothers and that outcomes were related to the integration policies of their host country [33]. Although there may be a 'healthy migrant effect' in some cases this may lead to immigrant women being medically lower-risk, restricted access to healthcare, poor financial stability and/ or low social support may lead to poorer outcomes [34, 35]. This is supported by studies reporting that migrant women face numerous barriers to healthcare including communication and language barriers and a lack of information regarding expected levels of antenatal care [36]. In addition, socio-economic factors amongst migrant women relate to adverse outcomes [37], as also seen in native-born women in high-income countries [38]. 


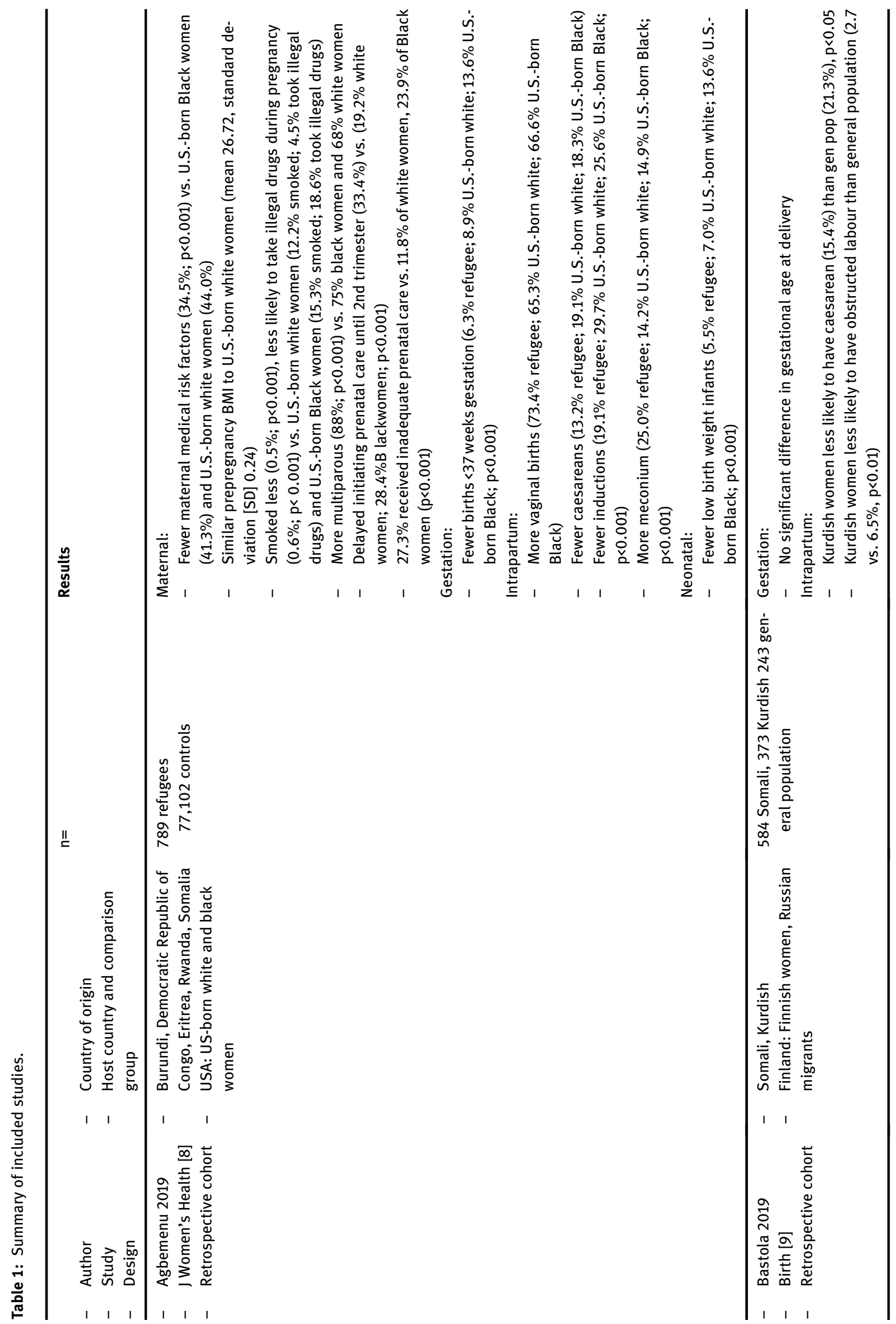




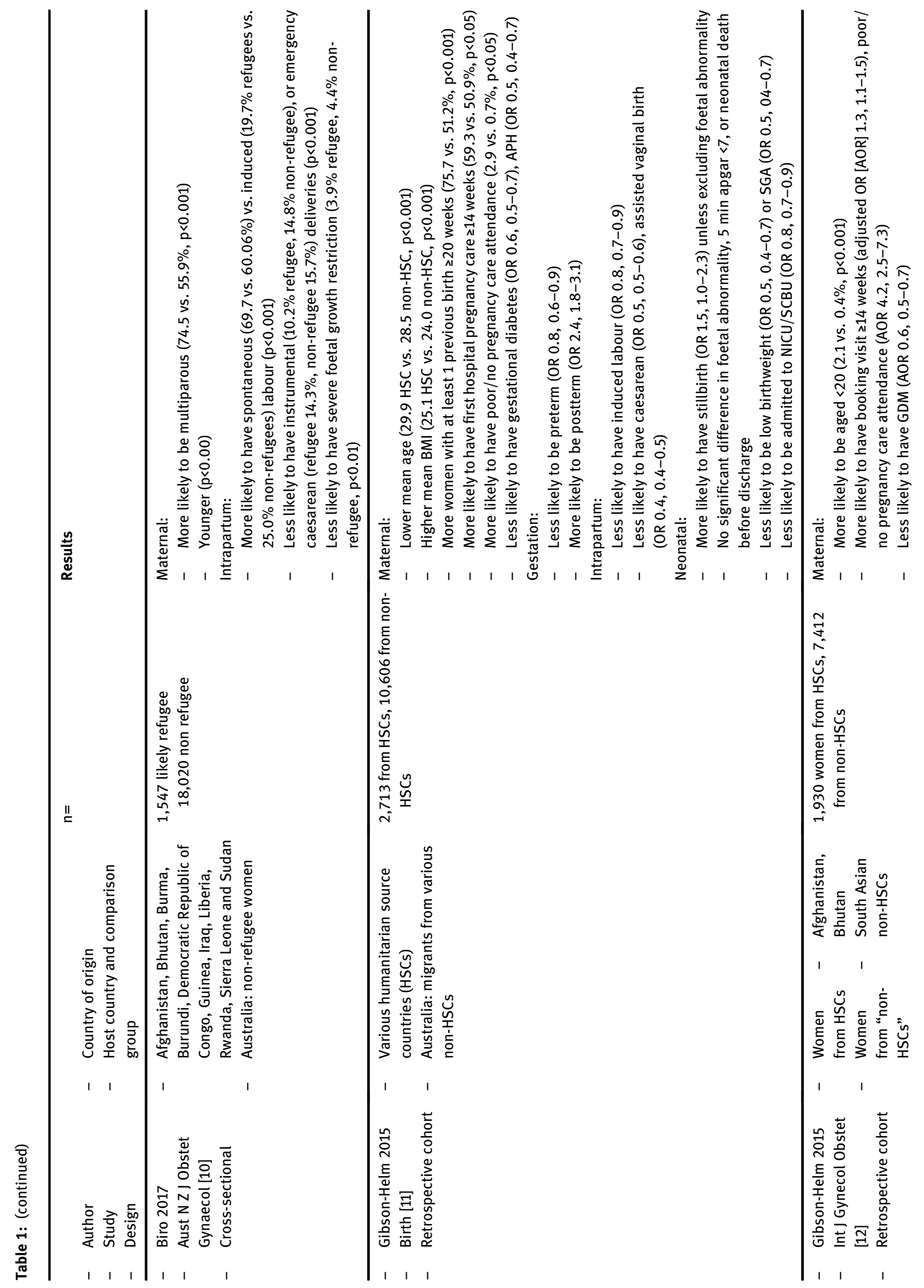




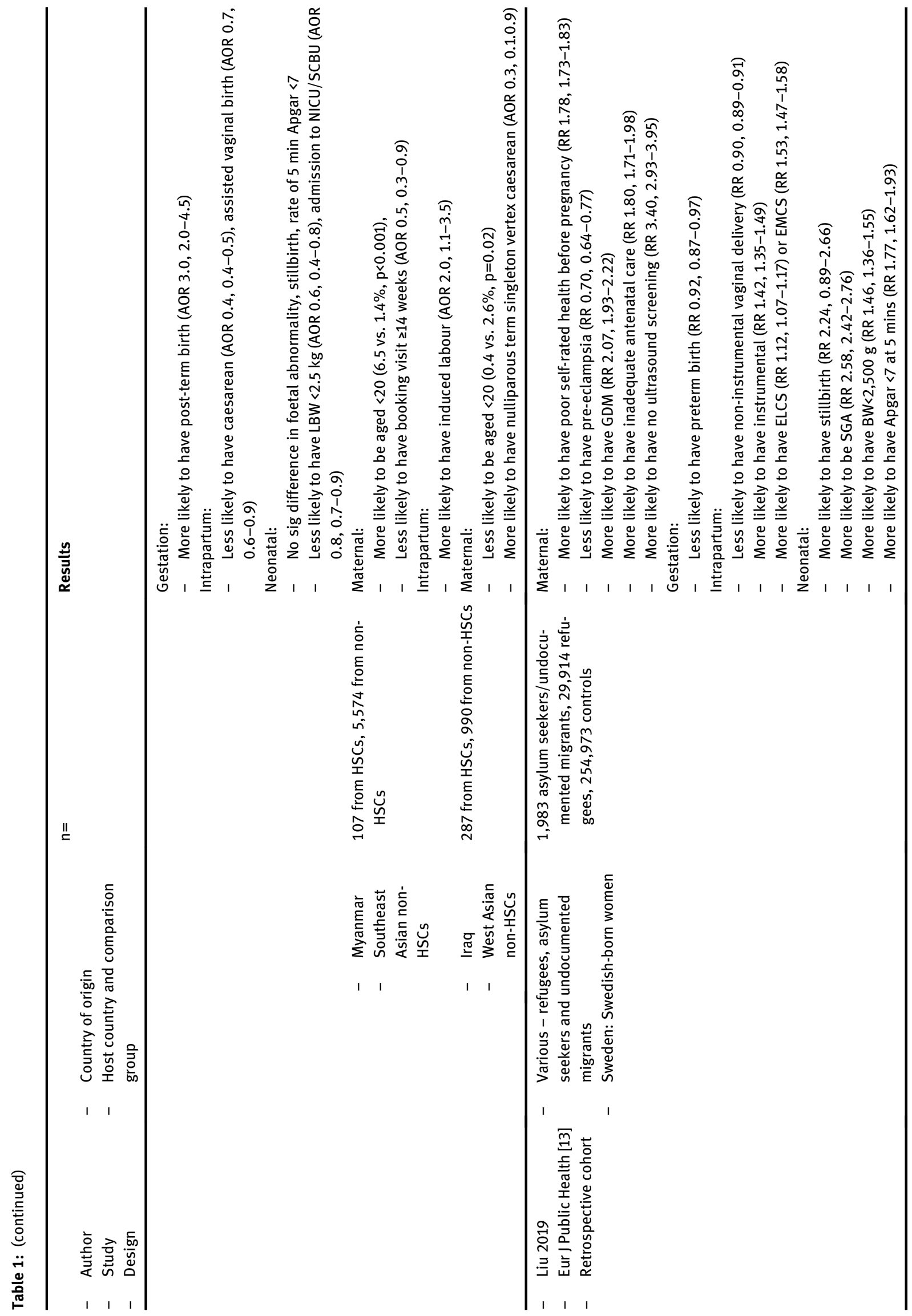




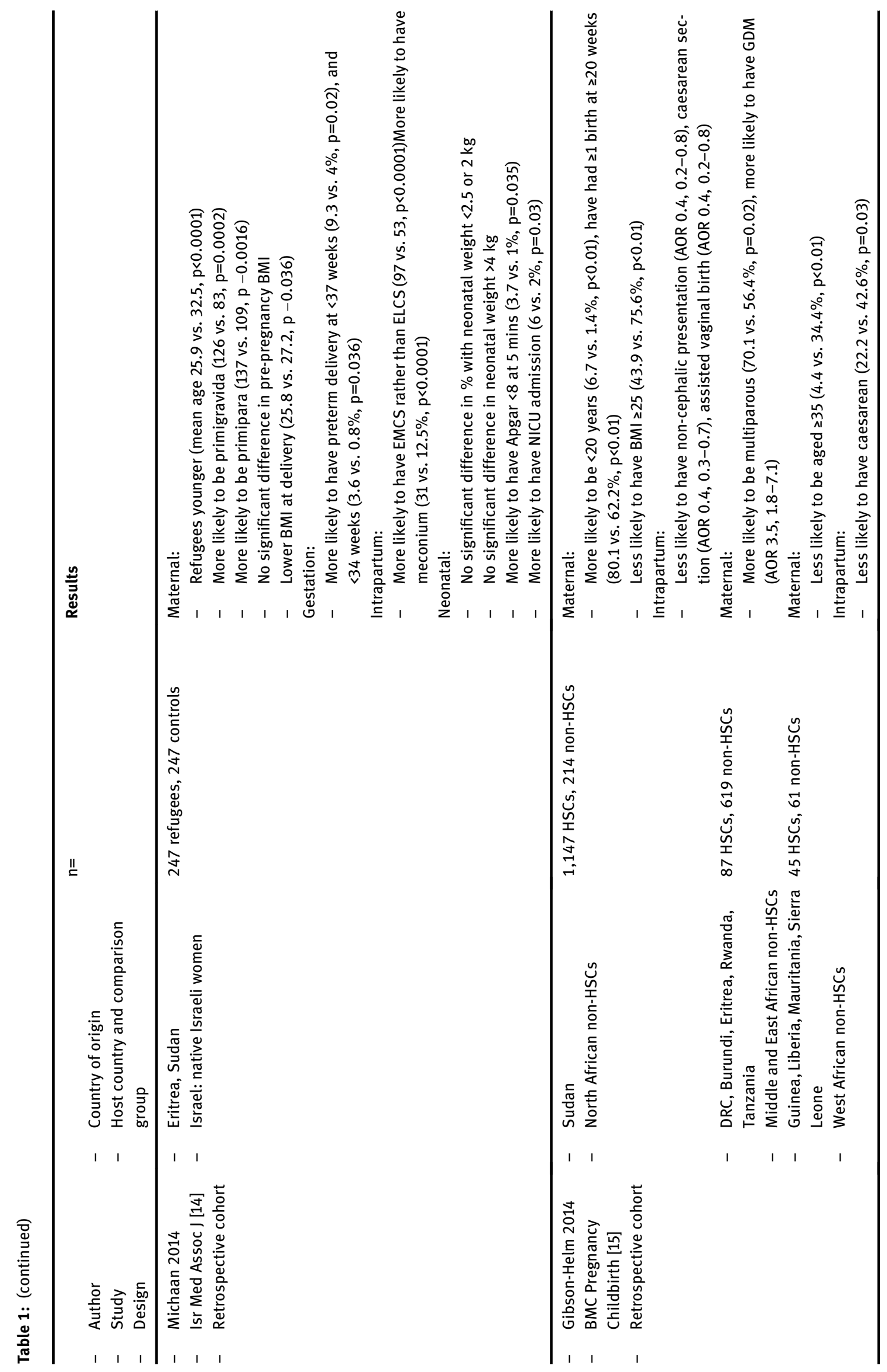




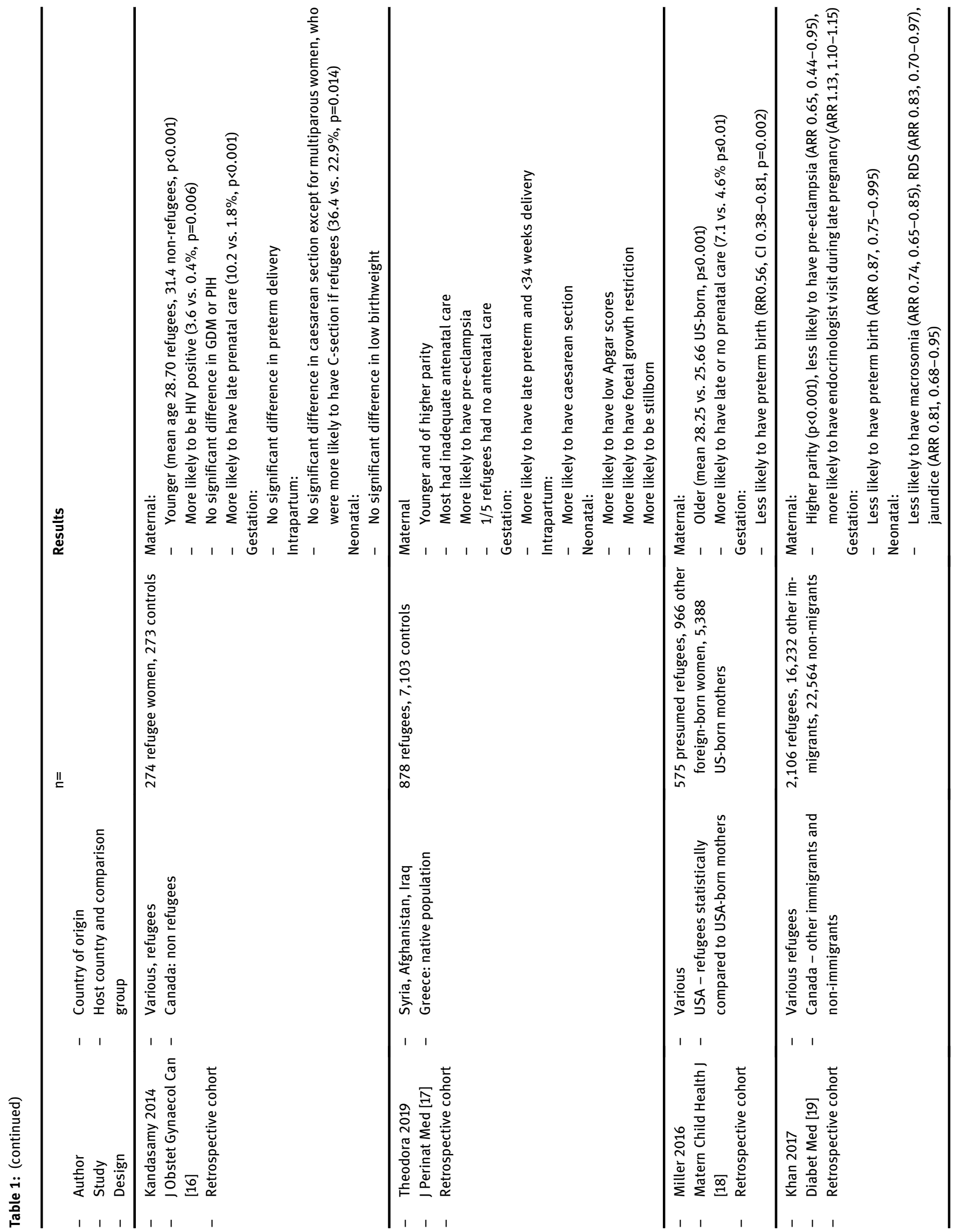




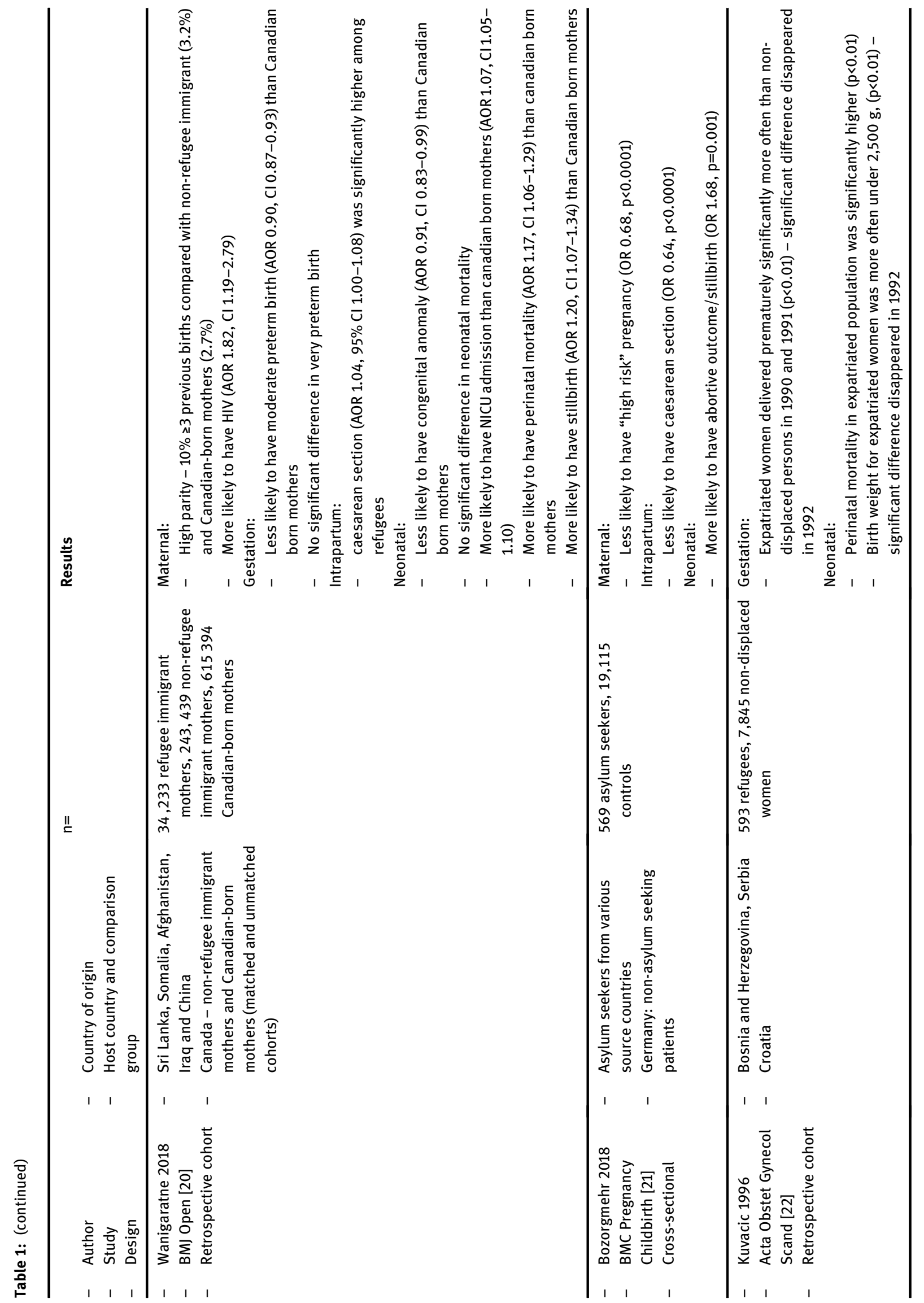




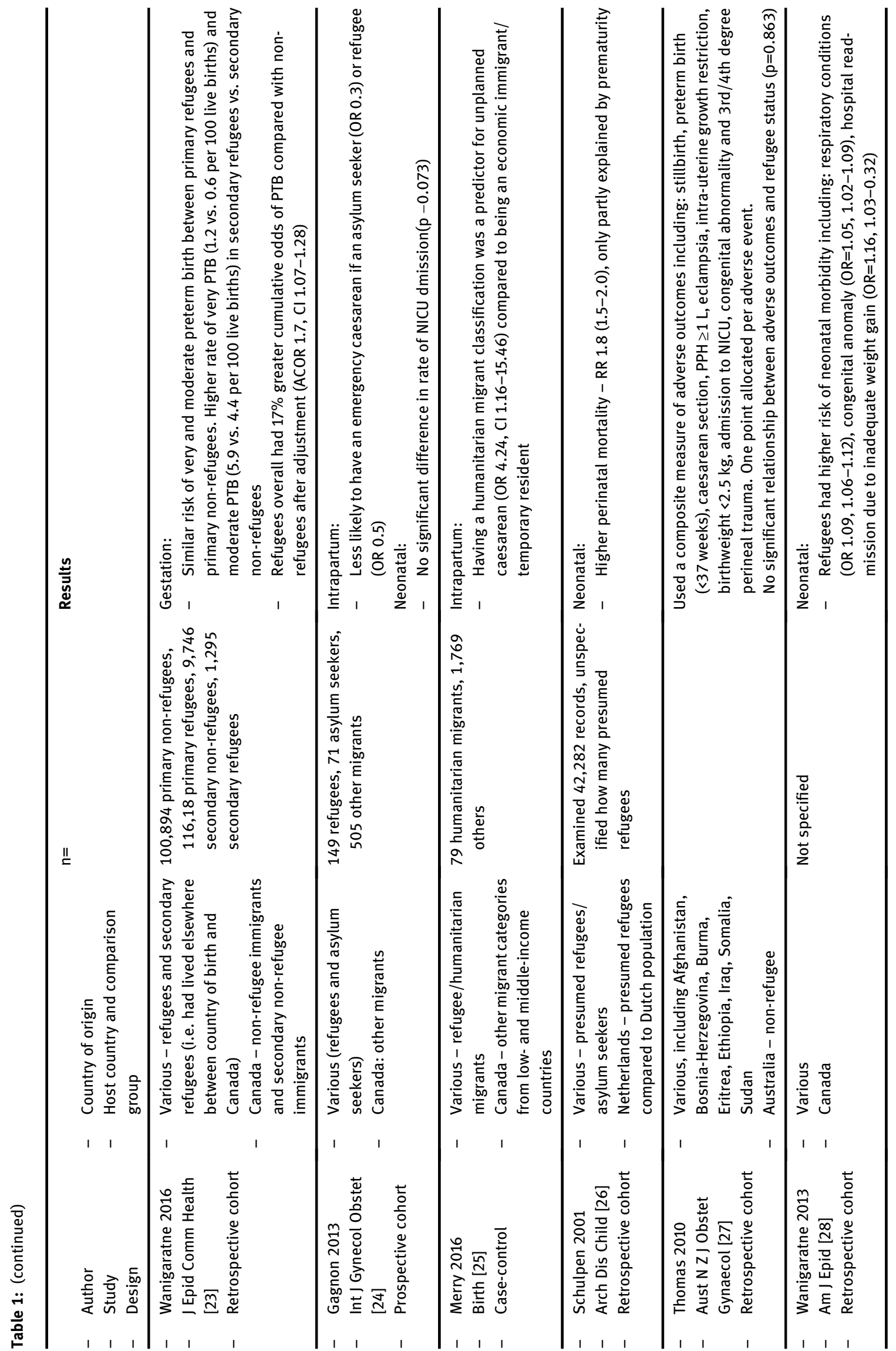




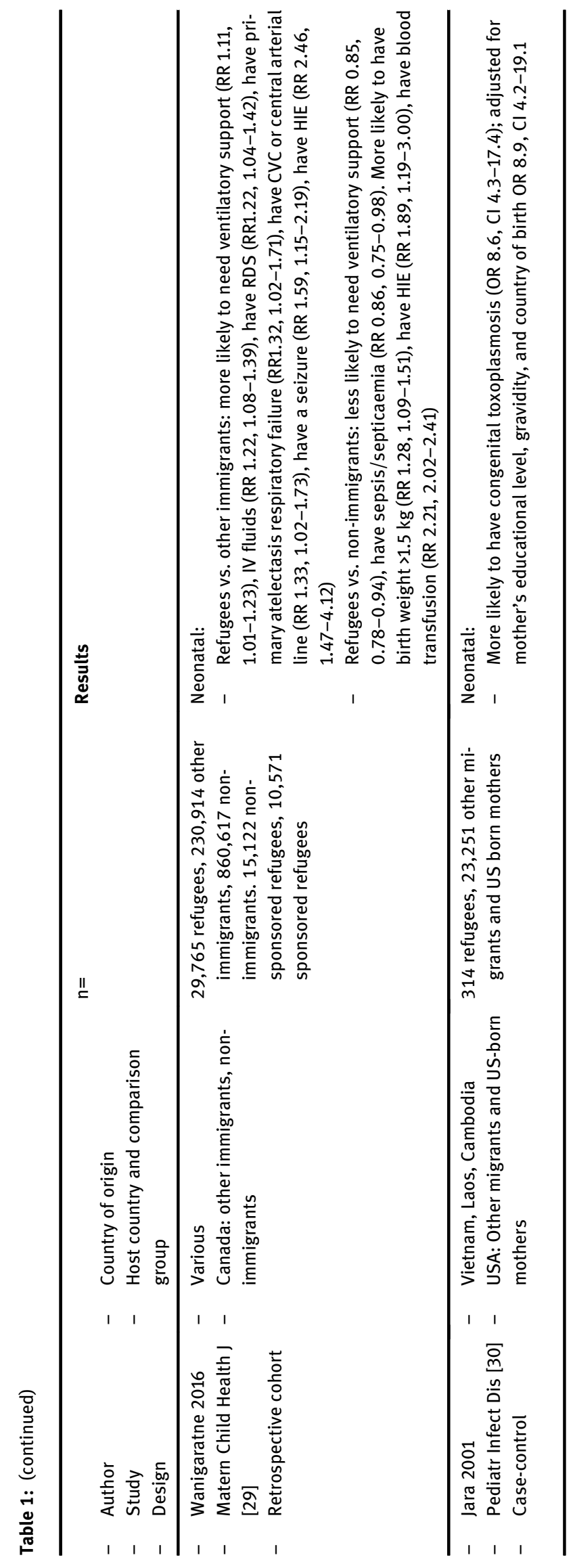


This review has a number of strengths, but some limitations. A number of large, population-based studies which included hundreds of refugee and asylum-seeking women were identified. The design of the cross-sectional and retrospective cohort studies limited the potential for bias, as the data included was quantitative and objective such as gestational age at delivery, method of delivery, and birthweight. Some studies directly compared refugees to other categories of migrants, reducing the potentially confounding effects of ethnicity and childhood access to healthcare. Limitations of the study include there are relatively few studies examining outcomes for refugee only, as opposed to broader groups of migrants. As we report, outcomes can differ significantly between refugees and non-refugees who have migrated from the same region to the same host country [11, 12, 15], as such findings of studies that included economic migrants cannot necessarily be generalised to refugees. Additionally, despite the inclusion of 26 studies, only 10 host countries were represented. This is a small fraction of high-income countries and the heterogeneous healthcare systems between countries may produce significantly different outcomes for socially disadvantaged mothers. The inclusion of a small fraction of high-income countries may reflect the small proportion (2.7\% in 2017) of global refugees and asylum seekers who are hosted in highincome countries compared to low- or middle-income countries [39]. The findings of this review that there is poor uptake of antenatal care and higher neonatal morbidity - may not be generalisable to all high-income countries, as some may have different payment structures and access provisions that can exacerbate or mitigate the barriers in immigrant access to healthcare.

\section{Conclusions}

Infants born to refugee and asylum-seeking women are more likely to be stillborn or to suffer increased neonatal morbidity in comparison to economic migrants and nativeborn women in high-income countries. This was despite refugee women being of medically low risk, with fewer pregnancy-related complications and has fewer instrumental or operative deliveries. They were, however, more likely to have poor, late or no access to antenatal care, which may explain the poorer neonatal outcomes.

Research funding: The research was supported by the National Institute for Health Research (NIHR) Biomedical Research Centre based at Guy's and St Thomas' NHS Foundation Trust and King's College London. The views expressed are those of the authors and not necessarily those of the NHS, the NIHR or the Department of Health.
Author contributions: SS and AG were responsible for the conception and design of the study; SS and EW were responsible for the literature review; SS, EW AG were responsible for drafting the article and revising it critically for important intellectual content. All authors have accepted responsibility for the entire content of this manuscript and approved its submission.

Competing interests: Authors state no conflict of interest. Ethical approval: Ethical approval was not required for this study as it was a literature review.

\section{References}

1. United Nations High. Commissioner for refugees (UNHCR). Figures at a glance [Internet]; 2019. Available from: https://www. unhcr.org/uk/figures-at-a-glance.html [Accessed 15 Mar 2020].

2. United Nations Women. Women refugees and migrants [Internet]; 2020. Available from: https://www.unwomen.org/en/news/infocus/women-refugees-and-migrants [Accessed 15 Mar 2020].

3. Women's Refugee Commission. Facts and figures [Internet]; 2020. Available from: https://www.womensrefugeecommission. org/empower/resources/practitioners-forum/facts-and-figures [Accessed 15 Mar 2020].

4. World Health Organization. Improving the health care of pregnant refugee and migrant women and newborn children. Technical Guidance. Copenhagen: WHO Regional Office for Europe; 2018, vol 52.

5. DeJong J, Ghattas H, Bashour H, Mourtada R, Akik C, ReeseMasterson A. Reproductive, maternal, neonatal and child health in conflict: a case study on Syria using countdown indicators. BMJ Glob Health 2017;2:e000302.

6. World Bank. Refugee population by country or territory of asylum [Internet]; 2019. Available from: https://data.worldbank.org/ indicator/SM.POP.REFG.

7. World Bank. World Bank Country and lending groups [Internet]; 2020. Available from: https://datahelpdesk.worldbank.org/ knowledgebase/articles/906519-world-bank-country-andlending-groups [Accessed 15 Mar 2020].

8. Agbemenu K, Auerbach S, Murshid NS, Shelton J, AmutahOnukagha N. Reproductive health outcomes in African refugee women: a comparative study. J Womens Health 2019;28:785-93.

9. Bastola K, Koponen P, Harkanen T, Luoto R, Gissler M, Kinnunen T. Delivery and its complications among women of Somali, Kurdish, and Russian origin, and women in the general population in Finland. Birth 2019;46:35-41.

10. Biro MA, East C. Poorer detection rates of severe fetal growth restriction in women of likely refugee background: a case for refocusing pregnancy care. Aust N Z J Obstet Gynaecol 2017;57: 186-92.

11. Gibson-Helm M, Teede H, Cheng IH, Block AA, Knight M, East CE, et al. Maternal health and pregnancy outcomes comparing migrant women born in humanitarian and nonhumanitarian source countries: a retrospective, observational study. Birth 2015;42:116-24.

12. Gibson-Helm M, Boyle J, Cheng I-H, East C, Knight M, Teede H. Maternal health and pregnancy outcomes among women of 
refugee background from Asian countries. Int J Gynecol Obstet 2015;129:146-51.

13. Liu C, Ahlberg M, Hjern A, Stephansson O. Perinatal health of refugee and asylum-seeking women in Sweden 2014-17: a register-based cohort study. Eur J Public Health 2019;19: 1048-55.

14. Michaan N, Gil Y, Amzalag S, Laskov I, Lessing J, Many A, et al. Perinatal outcome and financial impact of Eritrean and Sudanese refugees delivered in a tertiary hospital in Tel Aviv, Israel. Isr Med Assoc J 2014;16:371-4.

15. Gibson-Helm M, Teede H, Block A, Knight M, East C, Wallace EM, et al. Maternal health and pregnancy outcomes among women of refugee background from African countries: a retrospective, observational study in Australia. BMC Pregnancy Childbirth 2014; 14:392.

16. Kandasamy T, Cherniak R, Shah R, Yudin MH, Spitzer R. Obstetric risks and outcomes of refugee women at a single centre in Toronto. J Obstet Gynaecol Can 2014;36:296-302.

17. Theodora M, Antsaklis P, Michala L, Lolos M, Kalambalikis A, Koutroumanis $P$, et al. Perinatal outcomes among immigrants and refugees in comparison with Greek population in a tertiary university hospital in Greece. J Perinat Med 2019;47:eA126-326.

18. Miller LS, Robinson JA, Cibula DA. Healthy immigrant effect: preterm births among immigrants and refugees in Syracuse, NY. Matern Child Health J 2016;20:484-93.

19. Khan S, Yao Z, Shah B. Gestational diabetes care and outcomes for refugee women: a population-based cohort study. Diabet Med 2017;34:1608-14.

20. Wanigaratne S, Shakya Y, Gagnon AJ, Cole DC, Rashid M, Blake J, et al. Refugee maternal and perinatal health in Ontario, Canada: a retrospective population-based study. BMJ Open 2018;8:1-11.

21. Bozorgmehr K, Biddle L, Preussler S, Mueller A, Szecsenyi J. Differences in pregnancy outcomes and obstetric care between asylum seeking and resident women: a cross-sectional study in a German federal state, 2010-2016. BMC Pregnancy Childbirth 2018;18:417.

22. Kuvacic I, Skrablin S, Hodzic D, Milkovic G. Possible influence of expatriation on perinatal outcome. Acta Obstet Gynecol Scand 1996;75:367-71.

23. Wanigaratne S, Cole DC, Bassil K, Hyman I, Moineddin R, Urquia $M L$. The influence of refugee status and secondary migration on preterm birth. J Epidemiol Community Health 2016;70:622-8.

24. Gagnon A, Merry L, Haase K. Predictors of emergency cesarean delivery among international migrant women in Canada. Int J Gynecol Obstet 2013;121:270-4.

25. Merry L, Semenic S, Gyorkos T, Fraser W, Gagnon AJ. Predictors of unplanned cesareans among low-risk migrant women from lowand middle-income countries living in Montreal, Canada. Birth 2016;43:209-19.
26. Schulpen T, van Steenbergen J, van Driel H. Influences of ethnicity on perinatal and child mortality in the Netherlands. Arch Dis Child 2001;84:222-6.

27. Thomas PE, Beckmann M, Gibbons K. The effect of cultural and linguistic diversity on pregnancy outcome. Aust N Z J Obstet Gynaecol 2010;50:419-22.

28. Wanigaratne S, Urquia M. Risk of neonatal morbidity among infants born to refugee and non-refugee immigrant women in Ontario, Canada. Am J Epidemiol 2013;177:S146.

29. Wanigaratne S, Cole DC, Bassil K, Hyman I, Moineddin R, Shakya $Y$, et al. Severe neonatal morbidity among births to refugee women. Matern Child Health J 2016;20:2189-98.

30. Jara M, Hsu HW, Eaton RB, Demaria A Jr. Epidemiology of congenital toxoplasmosis identified by population-based newborn screening in Massachusetts. Pediatr Infect Dis J 2001; 20:1132-5.

31. Margioula-Siarkou C, Petousis S, Kalogiannidis I, Dagklis T, Traianos V, Goutzioulis M, et al. Immigrants present improved obstetric and neonatal outcomes compared to native women. A Northern Greek population analysis. J Immigr Minor Heal 2013;15: 249-54.

32. Singh GK, Stella MY. Adverse pregnancy outcomes: differences between US- and foreign-born women in major US racial and ethnic groups. Am J Public Health 1996;86: 837-43.

33. Bollini P, Pampallona S, Wanner P, Kupelnick B. Pregnancy outcome of migrant women and integration policy: a systematic review of the international literature. Soc Sci Med 2009;68: 452-61.

34. Langlois EV., Haines A, Tomson G, Ghaffar A. Refugees: towards better access to health-care services. Lancet 2016; 387:319-21.

35. Gushulak BD, Pottie K, Hatcher Roberts J, Torres S, DesMeules M. Migration and health in Canada: health in the global village. CMAJ 2011;183:E952-8.

36. Bollini P, Stotzer U, Wanner P. Pregnancy outcomes and migration in Switzerland: results from a focus group study. Int J Public Health 2007;52:78-86.

37. Campbell OM, Graham WJ. Strategies for reducing maternal mortality: getting on with what works. Lancet 2006;368: 1284-99.

38. Raatikainen K, Heiskanen N, Heinonen S. Under-attending free antenatal care is associated with adverse pregnancy outcomes. BMC Public Health 2007;7:1-8.

39. United Nations - Department of Economic and Social Affairs. International migration 2019 report. Handbook of sociology and human rights. New York, USA: International Population Division; 2019. 300-7 pp. (ST/ESA/SER.A/438). 